\title{
Review: evidence for the effectiveness of attention training in schizophrenia is inconclusive
}

Suslow T, Schonauer K, Arolt V. Attention training in the cognitive rehabilitation of schizophrenic patients: a review of efficacy studies. Acta Psychiatr Scand 2001 Jan;103:15-23.

\author{
QUESTION: In patients with schizophrenia, how effective is attention and cognitive \\ training in improving attentional functioning?
}

\section{Data sources}

Studies were identified by searching Medline (1966 to May 1999), Current Contents (1985 to May 1999), PsycLIT (1981 to March 1999), and Psyndex (1977 to March 1999), and by scanning the reference lists of key papers.

\section{Study selection}

Studies were selected if they evaluated the effectiveness of computer based or non-computer based attention training in patients with schizophrenia. Studies were excluded if the focus of the cognitive rehabilitation was on cognitive functions other than attention, there was no control group, there was a poor report of performance data, there were no external measures of outcome, or the study was not peer reviewed.

\section{Data extraction}

Data were extracted on type and duration of treatment, chronicity and subtype of disorder, and main outcome measures. One tailed effect sizes were calculated to evaluate before-after treatment changes in performance on external outcome measures.

\section{Main results}

9 studies met the selection criteria, 4 non-computer based treatments and 5 computer based. The number of participants in the experimental groups was small (median 17, range 10-40) and the duration of training was short (median $6 \mathrm{wk}$, range 3-12 wk). 2 studies evaluating the effectiveness of computer based attentional training found a statistically significant improvement of attentional functioning in the treatment groups compared with the control groups (effect sizes for the treatment groups ranged from 0.18 to 0.79 on various outcome measures $v-0.08$ to 0.12 for the control groups). 3 other studies (1 non-computer based and 2 computer based) found a differential training effect for 1 outcome variable (total letters processed, modality shift reaction time test, or Continuous Performance Test score) all favouring the treatment group.

For most applied outcomes measures (19 of 35) no performance improvement was observed after treatment. In all cases effect sizes were small.

\section{Conclusion}

There is inconclusive evidence that attention training is effective in schizophrenia.

\section{COMMENTARY}

Deficits in attention are likely to reflect an enduring vulnerability to the development of schizophrenia. Experimental evidence shows that these problems are present in individuals who are either actively symptomatic, remitted, or at high risk of developing schizophrenia. Indeed attention deficits are associated with impaired social functioning. Therefore, the rationale for providing training to ameliorate these deficits is to reduce vulnerability to further acute episodes, and improve social and cognitive functioning.

This review by Suslow et al shows that attention training, delivered over relatively brief time scales, does not produce reliable improvements in attentional processes. Any effect of attention training on social skills, social functioning, or relapse are not reported. Does this mean that we should abandon attention training? Attention problems in schizophrenia exist alongside a range of other cognitive processing problems such as memory and executive functioning. Therefore, attention training should be seen as a component of a more comprehensive approach to the remediation of cognitive deficits. Indeed, 2 of the 9 studies reviewed were evaluations of subprogrammes belonging to more comprehensive approaches. In addition, Suslow et al's rationale for focusing their review on attention training is that attention processes are the building blocks necessary for learning and favourable outcomes. However, it could equally be argued that attention deficits are secondary to problems in executive functioning (cognitive flexibility and planning). Therefore, targeting attention on its own does not necessarily address the key underlying cognitive deficits. There is some evidence that targeting executive functioning deficits is effective in remediating cognitive deficits, and that improved cognitive flexibility improves social functioning. ${ }^{1}$ It can therefore be argued that the targeting of attention deficits as part of more comprehensive programmes is a first step to introducing individuals to treatments aimed at improving cognitive skills and other areas of functioning. However, a recent systematic review in this area reports inconclusive results. ${ }^{2}$

Andrew Gumley, BA, MAppSci University of Stirling Stirling, UK

1 Wykes T, Reeder C, Corner J, et al. The effects of neurocognitive remediation on executive processing in patients with schizophrenia. Schizophr Bull 1999;25:291-307.

2 Hayes RL, McGrath JJ. Cognitive rehabilitation for people with schizophrenia and related conditions. Cochrane Database Syst Rev 2001;(1):CD000968. 